\title{
The Carina-Near Moving Group
}

\author{
B. Zuckerman \\ Dept. of Physics \& Astronomy and NASA Astrobiology Institute \\ University of California, Los Angeles \\ Los Angeles, CA 90095-1562, USA, ben@astro.ucla.edu \\ M. S. Bessell \\ Research School of Astronomy and Astrophysics \\ Institute of Advanced Studies \\ The Australian National University, ACT 2611, Australia, bessell@mso.anu.edu.au \\ Inseok Song, S. Kim \\ Gemini Observatory \\ 670 North A'ohoku Place \\ Hilo,HI96720,USA, song@gemini.edu,skim@gemini.edu
}

\begin{abstract}
We identify a group of $\sim 20$ co-moving, mostly southern hemisphere, $\sim 200 \mathrm{Myr}$ old stars near Earth. Of the stars likely to be members of this Carina-Near Moving Group, in either its nucleus ( 30 pc from Earth) or surrounding stream, all but 3 are plausible members of a multiple star system. The nucleus is (coincidentally) located quite close to the nucleus of the AB Doradus moving group notwithstanding that the two groups have substantially different ages and Galactic space motions, UVW.
\end{abstract}

Subject headings: open clusters and associations: individual (Carina-Near Moving Group) — stars: pre-main-sequence — stars: kinematics — stars: evolution

\section{Introduction}

During the past decade various young stellar associations and clusters have been identified within 100 pc of the Sun (see Zuckerman \& Song 2004, for a review). These comoving groups can illuminate our understanding of the properties of low and intermediate mass stars at times that correspond to the long-ago era when planet building and heavy bombardment 
were occurring in our Solar System. The Spitzer Infrared Observatory is targeting virtually all these young stars to determine if they are surrounded by dusty debris disks. And the stars are being observed at near-infrared wavelengths with the Hubble Space Telescope and with adaptive optics systems on most of the world's largest telescopes; the goal is to detect thermal emission from young massive planets.

In the present Letter we add another example - the Carina-Near Moving Group - to the growing menagerie of nearby young associations. The nucleus - consisting of stars in the relatively narrow range of spectral type F through K0 of which HR 3070 (= HIP 38160) is the earliest spectral type and the brightest - is only $\sim 30$ pc from Earth (Table 1). Some of the surrounding stream stars, whose group membership is therefore less secure than those in the nucleus, are even closer to Earth; for example, GJ 358 is within 10 pc. Based primarily on lithium abundance and X-ray flux (see Section 3), we estimate the group to be somewhat older than the Pleiades.

Some nuclear stars in our proposed group were initially suggested as co-moving by Eggen (1988). Later, Makarov \& Urban (2000, hereafter MU2000) argued that they are part of a spatially very extensive, X-ray bright, moving group they dubbed "Carina-Vela". However, other than X-ray luminosity, their choice of group membership was predicated entirely on kinematics, while lacking even radial velocity determinations. Our past experience (Song et al. 2002) with stars proposed (Makarov \& Fabricius 2001) as members of the TW Hydrae Association, demonstrated that a purely convergent-point kinematic approach is insufficient to reliably pinpoint members of nearby young associations. Nonetheless, in the 5 years following publication of their paper, neither Makarov \& Urban nor any other astronomer has verified or denied their kinematic model. For example, both Cutispoto et al. (2002) and Wichmann et al. (2003) studied group member HIP 37563 without referencing the MU2000 paper. Indeed, unware of the MU2000 paper, we independently stumbled on the nearer (to Earth) members of their proposed association while pursuing our research program of identification of young nearby stars. Apparently, in their young star program, Jensen et al. (2004) also find some overlap with the MU2000 sample. However, based on the few stars explicitly mentioned in the Jensen et al abstract, the stars in their program appear, on average, to be about 3 times more distant from Earth than stars in our Table 1.

Our investigations of radial velocities and youth indicators such as lithium abundance and $\mathrm{H} \alpha$ intensity are described in the following sections. The essence of our results may be summarized as follows: we confirm some of the MU2000 suggested members, deny others, and add some of our own. In addition, we think it is not helpful, and may even be wrong, to associate very nearby stars such as those listed in Table 1 with the much more distant stars that MU2000 place together into a single moving group. Specifically, as is evident from their 
Fig. 5, the nearest members of their "Carina-Vela" group are three times closer to Earth than their proposed more distant members, with essentially no stars at intermediate distances. In view of these large spatial separations, lack of evidence that Galactic space velocities $U V W$ are all similar, and lack of data on lithium abundances and activity indicators, at present we see no compelling reason to suppose the Carina-Vela group stars shared a common origin.

Table 1 of MU2000, "Candidate members of the Carina-Vela moving group", contains 58 entries most of which are not particularly near Earth. Of these 58, only 5 are included in our Table 1 as candidate members of the moving group of stars we are considering and one MU2000 candidate is a definite non-member of our proposed moving group. Rather than being spread out to distances $>100 \mathrm{pc}$ and with generally unknown $U V W$ (i.e. MU2000's Carina-Vela Group), our Table $1 \mathrm{~A}$ and B stars are characterized by proximity to Earth and rather tight constraints (a few $\mathrm{km} / \mathrm{s}$ ) on measured $U V W$ velocities. In addition, stars listed in our Table 1B are not in the Carina-Vela direction. Finally, based on our Figures 1 and 2 and the discussion in Section 3, we believe that the Carina-Near stars must be older than the Pleiades, whereas IC 2391 which belongs to the Carina-Vela moving group proposed by MU2000 is generally regarded as younger than the Pleiades. In view of these many differences, we designate the stars we are considering to be the "Carina-Near Moving Group".

\section{Observations}

In January 2006, we obtained spectra of Table 1 stars with the double-beam grating and echelle spectrographs on the two Nasmyth foci of the Australian National University's $2.3 \mathrm{~m}$ telescope at Siding Spring Observatory. The primary goal of these observations is the measurement of stellar radial velocity along with the equivalent widths of the $\mathrm{H} \alpha$ and Li $\lambda 6708$ lines. Radial velocity, in conjunction with proper motion and parallax, enables one to calculate the three-dimensional Galactic space motions U,V,W that are essential for identification of individual moving groups.

Entries for radial velocity, lithium, and $\mathrm{H} \alpha$ in Table 1 are a mix of our own measurements and those of others. U,V,W was calculated using weighted averages of proper motions from the PPM and Tycho catalogs and occasionally a third catalog and of radial velocities measured by us and by others. For stars that are obviously binaries based on their small separation in the plane of the sky, for example HIP 37918 and 37923, we used the weighted mean of their Hipparcos measured distances to calculate the UVWs listed in Table 1.

We have divided Table 1 into three parts: (A) nuclear members of the Carina-Near 
Moving Group, (B) suggested "stream" members associated with the Group, and (C) stars suggested in MU2000 as comoving but rejected by us as group members based on UVW. For moving groups with ages $<50 \mathrm{Myr}$, mid and late M-type stars located above the main sequence are probably the most reliable way to establish age (see, e.g., discussion and Fig. 2 in Zuckerman \& Song 2004). However, we could identify no stars noticeably above the main sequence among our proposed group members. The absence of such pre-main sequence stars is consistent with our $\sim 200 \mathrm{Myr}$ age estimate for the group (Section 3).

\section{Discussion}

Lacking any M-type stars that lie above the main sequence, we estimate the age of the Carina-Near group using lithium abundance plus activity indicators. Figure 1 displays Li $\lambda 6708$ equivalent width (EW) vs. spectral type of Carina-Near group stars overplotted on Fig. 3 from Zuckerman \& Song (2004). The Carina-Near stars are seen to lie near the bottom envelope of lithium EW for Pleiades stars. Figure 2 shows the X-ray luminosity of Carina-Near stars, normalized by their bolometric luminosities, overplotted on Fig. 4 of Zuckerman \& Song (2004). Again, the Carina-Near stars lie near the bottom envelope of Pleiades stars. Finally, we measured H $\alpha$ lines in early-M type stars HIP 47425 and 15844AB and $\mathrm{C}$ (all of which have $B-V \sim 1.5$ ), to be $-0.77,-0.14$ and +0.29 respectively where a negative sign denotes emission. Comparison with $\mathrm{H} \alpha$ line activity in stars with similar $B-V$, see Fig. 5 in Zuckerman \& Song (2004) and Fig. 5 in Gizis et al. (2002), suggests that these stars are young, but not dramatically so.

With adaptive optics imaging, Martín (2003) found that GJ 900 (HIP 116384), is a triple system. Martin notes that his referee, J. Stauffer, argues that the age of GJ900 is $100 \mathrm{Myr}$ or older based on its position in a $V$ vs $V-I$ color-magnitude diagram and comparison with low-mass members of the IC 2391, IC 2602, and Pleiades clusters.

Our measurements, as indicated in Figures 1 and 2, are consistent with Stauffer's assessment and we assign an age of $200 \pm 50 \mathrm{Myr}$ to the Carina-Near Moving Group.

A remarkable aspect of the stars listed in parts (A) and (B) of Table 1 is the dominance of multiple star systems. Only HIP 36414, 37635 and 47425 appear, at this time, to be likely single stars. The situation regarding HIP 37563 and 38160 is unclear. While HIP 37563 and 38160 might be single stars (they are fairly far from each other in the plane of the sky), it is possible that either one or both might comprise a very wide multiple system with binary star HIP 37918 \& 37923. Specifically, HIP 37563 and 38160 are each separated in the plane of the sky by about one light year from HIP 37918 \& 37923. If the separation along the line 
of sight is comparable (or less), then HIP 37563 or 38160 or both could, with 37918 \& 37923 , be a very wide triple or quadruple system.

In their Section 5, MU2000 comment that "surprisingly many" of their proposed CarinaVela group stars are members of binaries; this trait, they note, may be characteristic of stellar youth. If so, then such youthful affinity for multiplicity has been preserved for $\sim 200 \mathrm{Myr}$ in the Carina-Near group.

\section{Conclusions}

We have identified a group of comoving $\sim 200$ Myr old stars that partially surround the Sun. At least four of these stars are close enough to Earth to have been included in the original Gliese catalog of nearby stars (1969): GJ 140, 358, 900, and 907.1. Like all previously discovered and younger associations of stars within 100 pc of Earth - TW Hydrae, Tucana/Horologium, $\beta$ Pictoris, AB Doradus, $\eta$ Cha, Cha-Near - the nucleus of the Carina-Near Moving Group is located deep in the southern hemisphere. This nucleus, is (coincidentally) located quite close to the nucleus of the AB Doradus Moving Group notwithstanding that the two groups have substantially different ages and Galactic space motions, UVW: AB Dor $(-8,-27,-14)$; Carina-Near $(-26,-18,-2)$. Indeed, the U velocity of the Carina-Near group is substantially more negative than the $\mathrm{U}$ velocities of all the other groups listed here. In any event, identification of this nearby group of stars, many of which have masses similar to the Sun, extends the time-line covered by these other groups to older ages. As instrumentation capabilities improve, astronomers will be able to follow the planetary formation process over the age interval from 8 to $200 \mathrm{Myr}$.

We thank an anonymous referee and referee M.Schmitz for constructive comments.

\section{REFERENCES}

Bessell, M. S. 2000, PASP, 112, 961

Cutispoto, G., Pastori, L., Pasquini, L., de Medeiros, J. R., Tagliaferri, G., \& Andersen, J. 2002, A\&A, 384, 491

Eggen, O. J. 1988, Ap\&SS, 142, 145

García-Sánchez, J., et al. 2001, A\&A, 379, 634 
Gizis, J. E., Reid, I. N., \& Hawley, S. L. 2002, AJ, 123, 3356

Jensen, E. L. N., Schlesinger, K. J., \& Higby-Naquin, C. T. 2004, BAAS, 205

King, J. R., Villarreal, A. R., Soderblom, D. R., Gulliver, A. F., \& Adelman, S. J. 2003, AJ, 125,1980

Makarov, V. V. \& Fabricius, C. 2001, A\&A, 368, 866

Makarov, V. V. \& Urban, S. 2000, MNRAS, 317, 289

Martín, E. L. 2003, AJ, 126, 918

Song, I., Bessell, M. S., \& Zuckerman, B. 2002, A\&A, 385, 862

Wichmann, R., Schmitt, J. H. M. M., \& Hubrig, S. 2003, A\&广A, 399, 983

Zboril, M., Byrne, P. B., \& Rolleston, W. R. J. R. 1997, MNRAS, 284, 685

Zuckerman, B. \& Song, I. 2004, ARA\& A, 42, 685 
Table 1. The Carina-Near Moving Group

\begin{tabular}{|c|c|c|c|c|c|c|c|c|c|c|c|}
\hline HIP & $\begin{array}{l}\text { Other } \\
\text { Name }\end{array}$ & $\begin{array}{l}\text { R.A. } \\
(\mathrm{J} 2000)\end{array}$ & $\begin{array}{c}\text { Dec. } \\
(\mathrm{J} 2000)\end{array}$ & $\begin{array}{l}\text { Sp. } \\
\text { Type }\end{array}$ & $\begin{array}{c}\mathrm{V} \\
(\mathrm{mag})\end{array}$ & $\mathrm{B}-\mathrm{V}$ & $\begin{array}{c}\mathrm{D} \\
(\mathrm{pc})\end{array}$ & $\begin{array}{c}\mathrm{RV} \\
(\mathrm{km} / \mathrm{s})\end{array}$ & $\begin{array}{l}\mathrm{Lx} / \mathrm{Lbol} \\
\quad(\log )\end{array}$ & $\begin{array}{c}\mathrm{Li} \\
(\mathrm{m} \AA)\end{array}$ & $\begin{array}{c}\mathrm{U}, \mathrm{V}, \mathrm{W} \text { (uncertainty) } \\
(\mathrm{km} / \mathrm{s})\end{array}$ \\
\hline \multicolumn{12}{|c|}{ A. Members of the nucleus of the Carina-Near Moving Group } \\
\hline $35564 \mathrm{~A}$ & $\cdots$ & 072021.4 & -521841 & $\mathrm{~F} 2$ & 6.05 & 0.48 & 34.8 & $18.6 \pm 2$ & $-4.99^{\dagger}$ & $\ldots$ & $1.8-26.2-15.3-4.6\left(\begin{array}{lll}1.2 & 1.9 & 1.1\end{array}\right)$ \\
\hline 35564B & $\ldots$ & 072021.9 & -521833 & G0 & 6.60 & 0.58 & 34.8 & $\ldots$ & $-4.99^{\dagger}$ & 95 & $1.0 \quad \cdots$ \\
\hline 36414 & HD59704 & 072931.4 & -380721 & F7 & 7.75 & 0.52 & 52.5 & $28 \pm 2$ & -4.39 & 80 & $1.3-26.1-20.7-2.6\left(\begin{array}{llll}0.9 & 1.9 & 0.5\end{array}\right)$ \\
\hline 37563 & HD62850 & 074236.1 & -591751 & $\mathrm{G} 2 / 3$ & 7.19 & 0.62 & 33.3 & $17 \pm 1$ & -4.44 & 135 & $1.1-25.5-18.0-1.8\left(\begin{array}{llll}0.5 & 1.0 & 0.4\end{array}\right)$ \\
\hline 37635 & HD62848 & 074321.5 & -520951 & G0 & 6.70 & 0.55 & 30.2 & $20.5 \pm 0.5$ & -4.60 & 60 & $1.2-25.3-18.4-2.5\left(\begin{array}{lll}0.4 & 0.5 & 0.2\end{array}\right)$ \\
\hline 37918 & HD63581 & 074614.8 & -594851 & K0 & 8.15 & 0.77 & 36.2 & $17 \pm 1$ & $-4.34^{\dagger}$ & 110 & $0.9-26.0-18.1-2.4\left(\begin{array}{lll}1.8 & 1.0 & 0.4\end{array}\right)$ \\
\hline 37923 & HD63608 & 074617.0 & -594834 & K0 & 8.25 & 0.81 & 27.4 & $17 \pm 1$ & $-4.34^{\dagger}$ & 76 & $0.9-26.0-18.1-2.4\left(\begin{array}{lll}1.8 & 1.0 & 0.4\end{array}\right)$ \\
\hline 38160 & HR3070 & 074912.9 & -601701 & $\mathrm{~F} 1$ & 5.79 & 0.43 & 34.9 & $15 \pm 2$ & -5.38 & & $2.1-26.2-16.6-1.6\left(\begin{array}{llll}0.5 & 1.9 & 0.6\end{array}\right)$ \\
\hline \multicolumn{12}{|c|}{ B. Probable and possible members of the Carina-Near Stream } \\
\hline $15844 \mathrm{AB}$ & GJ140 & 032406.5 & +234706 & M1 & 10.4 & 1.45 & 19.8 & $19 \pm 2$ & -3.52 & 0 & $-0.1-24.3-17.0-6.0\left(\begin{array}{lll}1.9 & 2.1 & 0.9\end{array}\right)$ \\
\hline $15844 \mathrm{C}$ & GJ140C & 032412.7 & +234620 & M2.5 & 11.9 & $\cdots$ & 19.8 & $18 \pm 3$ & $\cdots$ & & $+0.3-23.3-15.0-4.6(2.72 .01 .4)$ \\
\hline 47425 & GJ358 & 093946.4 & -410403 & M2 & 10.77 & 1.52 & 9.5 & $18 \pm 3$ & -3.71 & 0 & $-0.8-28.8-17.8-0.9(0.53 .00 .5)$ \\
\hline 58240 & HD103742 & 115642.3 & -321605 & G3 & 7.64 & 0.65 & 34.9 & $6 \pm 0.4$ & $-4.46^{\dagger}$ & 111 & $1.1-21.2-17.1-3.1(3.31 .80 .9)$ \\
\hline 58241 & HD103743 & 115643.8 & -321603 & G3 & 7.81 & 0.70 & 29.2 & $6.7 \pm 0.3$ & $-4.46^{\dagger}$ & 110 & $1.1-21.0-17.6-2.8\left(\begin{array}{lll}3.3 & 1.8 & 0.9\end{array}\right)$ \\
\hline 60831 & HD108574 & 122804.4 & +444739 & $(\mathrm{~F} 7)$ & 7.40 & 0.56 & 39.2 & $-1.8 \pm 1$ & $-4.70^{\dagger}$ & 109 & $\cdots-28.5-17.8-4.4\left(\begin{array}{llll}2.0 & 1.2 & 1.0\end{array}\right)$ \\
\hline 60832 & HD108575 & 122804.8 & +444730 & (G0) & 7.97 & 0.66 & 42.4 & $\ldots$ & $-4.70^{\dagger}$ & 70 & $\cdots \quad-30.9-18.3-1.5(4.93 .02 .0)$ \\
\hline 116384 & GJ900 & 233500.3 & +013619 & M0 & 9.61 & 1.35 & 19.3 & $-10 \pm 2$ & -3.53 & $6 \pm 2$ & $\cdots-29.1-15.7+0.9\left(\begin{array}{llll}1.0 & 1.2 & 1.7\end{array}\right)$ \\
\hline 117410 & GJ907.1 & 234825.7 & -125915 & $\mathrm{~K} 8$ & 9.57 & 1.26 & 27.1 & $-8 \pm 2$ & -3.44 & $\cdots$ & $\cdots \quad-29.0-14.0+0.9\left(\begin{array}{lll}1.8 & 1.0 & 1.9\end{array}\right)$ \\
\hline \multicolumn{12}{|c|}{ C. Non-members } \\
\hline 37718 & HD63008 & 074412.5 & -502724 & $\mathrm{~F} 8$ & 6.64 & 0.53 & 30.8 & $8.5 \pm 0.3$ & $-4.60^{\dagger}$ & 67 & $1.3-26.4-4.4-6.2\left(\begin{array}{lll}0.3 & 0.3 & 0.2\end{array}\right)$ \\
\hline 37727 & HD63008B & 074416.5 & -502800 & G0 & 7.55 & 0.70 & 30.1 & $9.0 \pm 0.5$ & $-4.60^{\dagger}$ & 46 & $1.0-26.5-4.9-6.4\left(\begin{array}{llll}0.3 & 0.5 & 0.2)\end{array}\right.$ \\
\hline
\end{tabular}

Note. - $U V W$ are defined with respect to the Sun, with $U$ positive toward the Galactic Center, $V$ positive in the direction of Galactic rotation, and $W$ positive toward the north Galactic pole.

Average UVW for the nucleus of the Carina-Near Moving Group is $-25.9,-18.1,-2.3(\mathrm{~km} / \mathrm{sec})$.

HIP 35564B: We measured the radial velocity to be $59 \mathrm{~km} / \mathrm{sec}$ and $32 \mathrm{~km} / \mathrm{sec}$ in 2006 January and April, respectively; so this star may be a spectroscopic binary.

HIP 37563: We measured $125 \mathrm{~m} \AA$ for lithium $6708 \AA$. Wichmann et al (2003) and Cutispoto et al (2002) each measured $145 \mathrm{~m} \AA$. HIP 37718 \& 37727: parallax used for calculation of UVW is $32.8 \pm 0.4$ mas. Proper motion $=(-110.8 \pm 1.0,+143.0 \pm 1.0)$ mas $/ \mathrm{yr}$ HIP $37918 \&$ 37923: parallax used is $29.2 \pm 2.0$ mas. Proper motion $=(-58.0 \pm 1.5,+155 \pm 1.5) \mathrm{mas} / \mathrm{yr}$

HIP 47425 : García-Sánchez et al. (2001) list RV=142 $\pm 21 \mathrm{~km} / \mathrm{sec}$.

HIP 58240 \& 58241: parallax used is $31.45 \pm 4.5$ mas. Proper motion $=(-177.4 \pm 2.0,-5.3 \pm 2.0) \mathrm{mas} / \mathrm{yr}$ HIP 60831 \& 60832: listed spectral types are estimated from $V-K$ color.

HIP 116384: listed lithium $6708 \AA$ equivalent width is from Zboril et al. (1997). Triple system (Martín 2003).

HIP 117410: 1" Hipparcos binary.

$\dagger$ : ROSAT X-ray positional uncertainty circle covers both stars in the 4 indicated binary systems. To calculate fractional X-ray luminosity in each case, we "shared" the X-ray flux between the two stars. 


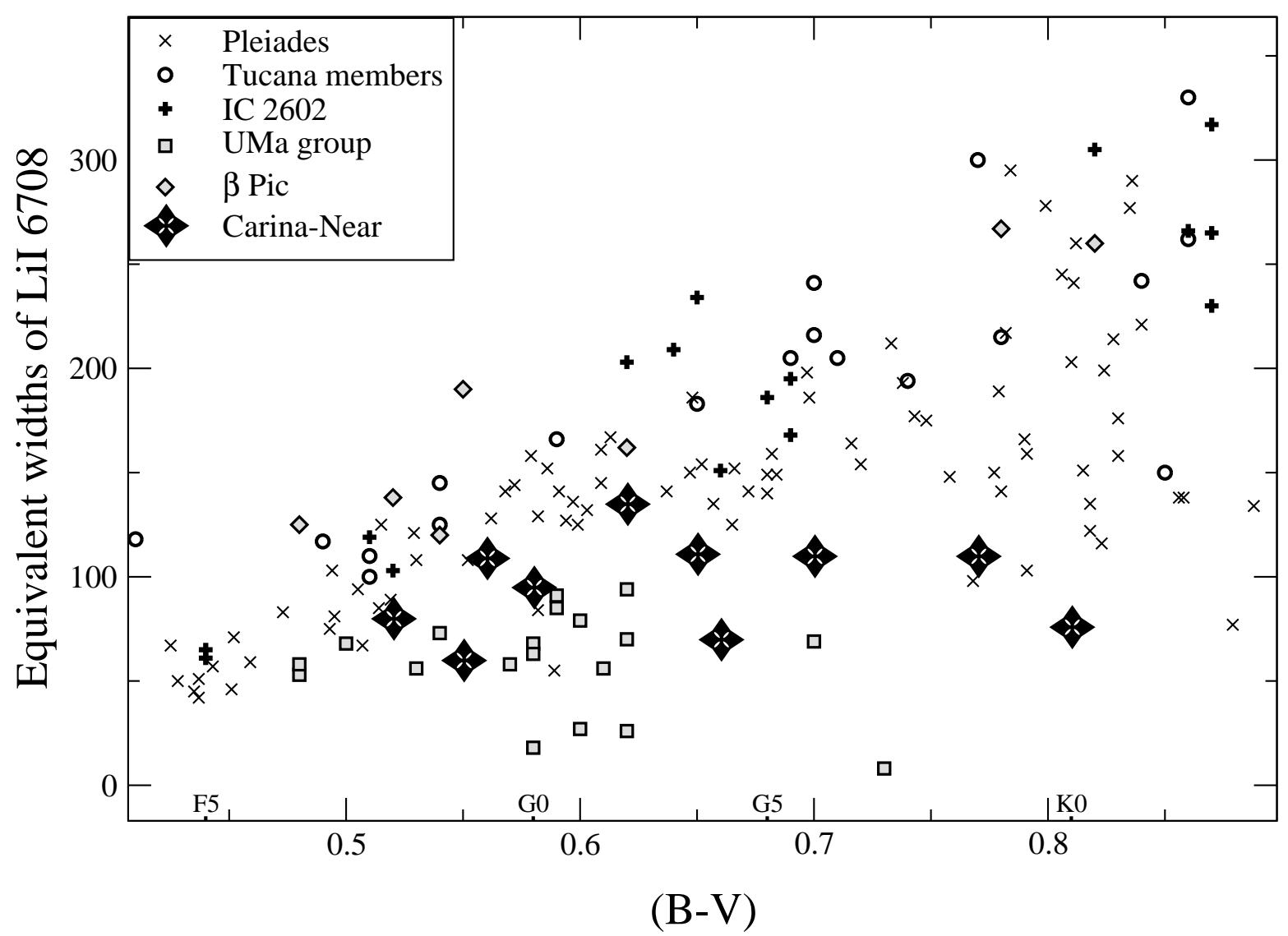

Fig. 1.- Equivalent width of Li I $6708 \AA$ as a function of $B-V$. Displayed equivalent widths are not corrected for possible contamination by Fe I6707.44 $\AA$, and measurement uncertainty of equivalent widths is $\sim 20 \mathrm{~m} \AA . B-V$ values for Carina-Near group stars are calculated from Tycho-2 $B_{T}$ and $V_{T}$ magnitudes using the relation given in Bessell (2000). 


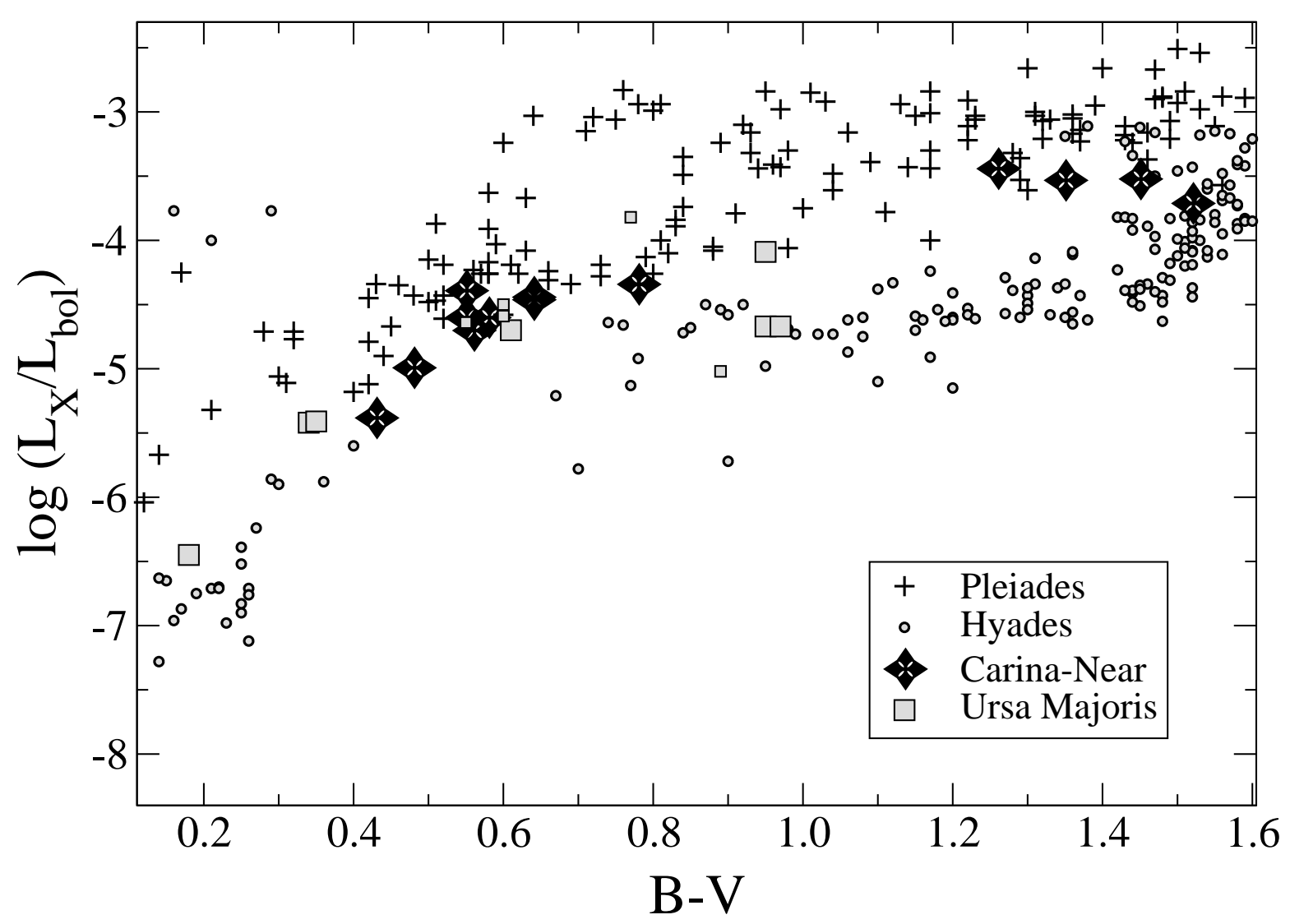

Fig. 2.- Ratio of X-ray to bolometric luminosity as a function $B-V$. $B-V$ values for Carina-Near group stars are calculated from Tycho-2 $B_{T}$ and $V_{T}$ magnitudes as in Figure 1. Ursa Majoris moving group (estimated age of $500 \pm 100 \mathrm{Myr}$; King et al. 2003) stars are plotted as square symbols (large squares for nucleus members). Based on the relative position in this plot of UMa and Hyades member stars, if the Hyades age is $\sim 600 \mathrm{Myr}$ as is usually quoted, then the UMa stars are probably $\sim 400 \mathrm{Myr}$ old. B-V color of a multiple system was calculated to reproduce single color for matching the composite Lx/Lbol value of the system. 